\title{
Robust Immune Response Induced by Schistosoma mansoni TSP-2 Antigen Coupled to Bacterial Outer Membrane Vesicles
}

\author{
Mayra M F Barbosa, (D) I,2 Alex I \\ Kanno,' Giovana C \\ Barazzone, (iD)' Dunia \\ Rodriguez,' Violeta \\ Pancakova, ',3 Monalisa Trentini,' \\ Eliana L Faquim-Mauro, (iD) ${ }^{4}$ \\ Amanda P Freitas, ${ }^{4}$ Mariana I \\ Khouri, (D) ${ }^{5}$ Jessica Lobo-Silva, ${ }^{5}$ \\ Viviane M Goncalves,' Rocilda P \\ F Schenkman,' Martha M \\ Tanizaki, ' Diana Boraschi, ${ }^{6-8}$ \\ Richard Malley, ${ }^{9}$ Leonardo P \\ Farias, ${ }^{5}$ Luciana C C Leite

\begin{abstract}
'Laboratório de Desenvolvimento de Vacinas, Instituto Butantan, São Paulo, Brazil; ${ }^{2}$ Programa de Pós-Graduação Interunidades em Biotecnologia, Universidade de São Paulo, São Paulo, Brazil; ${ }^{3}$ Université Claude Bernard Lyon I (UCBLI), Villeurbanne, 69100, France; ${ }^{4}$ Laboratório de Imunopatologia, Instituto Butantan, São Paulo, Brazil; ${ }^{5}$ Laboratório de Biomarcadores e Inflamação, Instituto Gonçalo Moniz, Fundação Oswaldo Cruz, Salvador, Brazil; 6 Istituto di Biochimica e Biologia Cellulare, Consiglio Nazionale delle Ricerche, Napoli, Italy; ${ }^{7}$ Stazione Zoologica Anton Dohrn, Napoli, Italy; ${ }^{8}$ Shenzhen Institute of Advanced Technologies (SIAT), Chinese Academy of Sciences (CAS), Shenzhen, China Division of Infectious Diseases, Boston Children's Hospital, Boston, MA, USA; 'Division of Infectious Diseases, Boston Children's Hospital, Boston, MA, USA
\end{abstract}

Correspondence: Luciana C C Leite Laboratório de Desenvolvimento de Vacinas, Instituto Butantan, Av. Vital Brasil, São Paulo, I500, SP, Brazil Tel +55-I I-2627-98I6

Email luciana.leite@butantan.gov.br
Purpose: The use of adjuvants can significantly strengthen a vaccine's efficacy. We sought to explore the immunization efficacy of bacterial outer membrane vesicles (OMVs) displaying the Schistosoma mansoni antigen, SmTSP-2, through a biotin-rhizavidin coupling approach. The rationale is to exploit the nanoparticulate structure and the adjuvant properties of OMVs to induce a robust antigen-specific immune response, in light of developing new vaccines against $S$. mansoni.

Materials and Methods: OMVs were obtained from Neisseria lactamica and conjugated with biotin. The recombinant SmTSP-2 in fusion with the biotin-binding protein rhizavidin (rRzvSmTSP-2) was produced in E. coli and coupled to biotinylated OMVs to generate an OMV complex displaying SmTSP-2 on the membrane surface (OMV:rSmTSP-2). Transmission electron microscopy (TEM) and dynamic light scattering analysis were used to determine particle charge and size. The immunogenicity of the vaccine complex was evaluated in C57BL/6 mice.

Results: The rRzvSmTSP-2 protein was successfully coupled to biotinylated OMVs and purified by size-exclusion chromatography. The OMV:rSmTSP-2 nanoparticles showed an average size of $200 \mathrm{~nm}$, with zeta potential around - $28 \mathrm{mV}$. Mouse Bone Marrow Dendritic Cells were activated by the nanoparticles as determined by increased expression of the costimulatory molecules CD40 and CD86, and the proinflammatory cytokines (TNF- $\alpha$, IL-6 and IL-12) or IL-10. Splenocytes of mice immunized with OMV:rSmTSP-2 nanoparticles reacted to an in vitro challenge with SmTSP-2 with an increased production of IL-6, IL-10 and IL-17 and displayed a higher number of CD4+ and CD8+ T lymphocytes expressing IFN- $\gamma$, IL-4 and IL-2, compared to mice immunized with the antigen alone. Immunization of mice with OMV:rSmTSP-2 induced a 100-fold increase in specific anti-SmTSP-2 IgG antibody titers, as compared to the group receiving the recombinant $\mathrm{rSmTSP}-2$ protein alone or even co-administered with unconjugated OMV.

Conclusion: Our results demonstrate that the SmTSP-2 antigen coupled with OMVs is highly immunogenic in mice, supporting the potential effectiveness of this platform for improved antigen delivery in novel vaccine strategies.

Keywords: Schistosoma mansoni, vaccine, TSP-2 antigen, outer membrane vesicles, OMV, biotin-avidin coupling, nanoparticle

\section{Introduction}

Many decades of research have been dedicated to developing vaccines against human parasitic diseases, such as schistosomiasis and malaria, considered by the World Health Organization (WHO) as the most devastating parasitic diseases for 
their socioeconomic and public health impact. ${ }^{1}$ Current chemotherapeutic approaches are highly effective in eliminating parasites, but do not protect from reinfection, and sequential mass chemotherapies are required. Therefore, an effective vaccine would be an invaluable asset to control these diseases. ${ }^{2,3}$

Over the last decades, many Schistosoma antigens have been evaluated as vaccine candidates, revealing Sm14, Sm29, Calpain and SmTSP-2 as the most promising candidates. $^{4-7}$ Among these, rSmTSP-2 provided high levels of protection against cercarial challenge in the mouse model. ${ }^{7}$ In humans, individuals resistant to infection after years of exposure displayed high levels of IgG1 and IgG3 antibodies against rSmTSP-2. Therefore, rSmTSP-2 holds great potential as a Schistosoma vaccine candidate. $^{7,8}$

The high level of protection achieved in mice was demonstrated when antigens were administered with Freund's adjuvant, which is not suitable for use in human vaccines. Recombinant proteins per se usually show low immunogenicity, a limitation that can be overcome with new formulations that include adjuvants, and with different antigen delivery systems. ${ }^{9-11}$ Classical approaches using subunit vaccines with different adjuvants, such as emulsions, cytokines and microbial products (eg, $\mathrm{CpG}$ and cholera toxin), did not increase protection significantly. ${ }^{1,12}$

A variety of antigen delivery systems can be used to enhance antigen recognition by the immune system. ${ }^{13,14}$ They include engineered viruses, bacteria, virus-like particles (VLPs) and outer membrane vesicles (OMVs). ${ }^{13}$ OMVs are naturally occurring nanoparticles, released by many microorganisms, in particular by Gram-negative bacteria, with a diameter of 30-200 nm. ${ }^{15-17}$ An example of OMV-based vaccine is the Bexsero ${ }^{\circledR}(4 \mathrm{CMenB})$ vaccine against Meningococcus B, composed of recombinant proteins selected by reverse vaccinology and including OMVs to increase immunogenicity and protection in humans. ${ }^{18}$ OMVs have been evaluated for decades in animals and humans, showing adjuvant properties, compatibility with different vaccine platforms and the ability to stimulate both humoral and cellular immune responses. ${ }^{15}$ OMVs, as nanoparticulate delivery systems for vaccination, have advantages in antigen presentation and adjuvanticity, due to their physical, chemical and biological characteristics, including size, surface charge and immunostimulatory properties. The exposure of antigens on the surface of some of these systems allows for binding with B cell receptors, leading to improved antibody-mediated responses. ${ }^{19,20}$

The recently developed Multiple Antigen Presenting System (MAPS) is an antigen delivery platform that can integrate several antigen components, including polysaccharides and proteins, in a macromolecular complex. The technique involves the association of pathogen-specific antigens, genetically in fusion with the biotin-binding protein rhizavidin, ${ }^{21}$ with a biotinylated backbone. ${ }^{22}$ This approach enables the generation of broad immune responses, with the induction of antibody, Th1 and Th17 responses, to multiple antigens. ${ }^{22}$

Here, we investigated the efficacy of OMVs as backbone for presentation of $S$. mansoni antigens and induction of antigen-specific immune responses. Biotinylated OMVs (OMV-B) were coupled with the SmTSP-2 antigen in fusion with rhizavidin (rRzvSmTSP-2). The OMV-B: rRzvSmTSP-2 complex (OMV:rSmTSP-2) was characterized by transmission electron microscopy (TEM) and dynamic light scattering and immunogenicity was evaluated in mice. Our results showed that the OMV:rSmTSP-2 complex induces very high levels of specific antibodies and a broad cellular immune response in mice, supporting future studies of this platform for improved antigen delivery.

\section{Materials and Methods}

\section{Ethics Statement}

This study was carried out in accordance with the guidelines of the Guide for Care and Use of Laboratory Animals from the Committee of the Sociedade Brasileira de Animais de Laboratório (SBAL). The experimental protocols for this work were approved by the Committee on Ethics of Animal Experiments of Butantan Institute (São Paulo, Brazil) (permit number: CEUA3314160715).

\section{OMV Production and Detoxification}

The Neisseria lactamica N.799/98 strain was gently provided by Instituto Adolfo Lutz (Núcleo de Coleção de Micro-organismos; São Paulo, Brazil) and used to obtain the OMVs. Bacterial samples maintained at $-80^{\circ} \mathrm{C}$ were thawed at $36^{\circ} \mathrm{C}$, and the bacterial suspension inoculated onto Müller-Hinton agar (Difco ${ }^{\mathrm{TM}}$; BD Biosciences, San Jose, CA, USA) and cultivated at $36^{\circ} \mathrm{C}$ in anaerobic conditions for $24 \mathrm{~h}$. The bacterial lawn formed on the agar was resuspended in $3 \mathrm{~mL}$ of the Catlin's modified MC2LAA-YE medium (Supplementary Table 1), ${ }^{23}$ 
inoculated in shaker flasks containing $30 \mathrm{~mL}$ of the same media, and was incubated at $36^{\circ} \mathrm{C}$ for $20 \mathrm{~h}$ at $200 \mathrm{rpm}$ (Amerex Instruments, Inc., Concord, CA, USA). The cultures were used to inoculate a second shaker flask with 100 mL media (300 mL Tunair Flask - full baffled: Merck KGaA, Darmstadt, Germany), which was further expanded to $1 \mathrm{~L}$ of MC2LAA-YE under the same conditions (modified from). ${ }^{24}$ The optical density was monitored until the end of the stationary phase when cultivation was interrupted, and the supernatant recovered after centrifugation at $3200 \times \mathrm{g}$. OMVs were recovered from the culture medium by ultracentrifugation (L8-M Ultracentrifuge; Beckman Coulter, Indianapolis, IN, USA) of $50 \mathrm{~mL}$ samples at $100,000 \times \mathrm{g}$ for $3 \mathrm{~h}$ and resuspension in $50 \mathrm{~mL}$ of PBS with $3 \%$ sucrose $(\mathrm{pH} 7.4)$. This procedure provided $105 \mathrm{mg}$ of OMV protein.

In order to reduce the LPS content, the OMV suspension $(50 \mathrm{~mL})$ was treated with $50 \mathrm{~mL}$ of a solution containing 1\% sodium deoxycholate (DOC) in $100 \mathrm{mM}$ Tris, 2 mM EDTA, $\mathrm{pH} 8.5$, and the mixture incubated at room temperature for $15 \mathrm{~min}$. To remove DOC and concentrate the sample, the OMV suspension was washed with $3 \%$ sucrose in PBS by tangential flow filtration, performed with a $\operatorname{Biomax}^{\circledR}$ membrane (Merck-Millipore, Burlington, MA, USA) with a $100 \mathrm{kDa}$ cut-off in LabScale $^{\mathrm{TM}}$ TFF System (Merck-Millipore). ${ }^{24}$ The total protein concentration of the OMVs was determined either by Bradford or by the Kjeldahl method, ${ }^{25}$ and the endotoxin concentration was determined by the gel-clot assay (Lonza Group Ltd., Basel, CH).

\section{Biotinylation of OMVs and rSmTSP-2 Binding}

The detoxified OMVs (dOMVs) $(\sim 25 \mathrm{mg})$ were conjugated with biotin using $10 \mathrm{mg}$ of the biotin derivative Amine-PEG3-Biotin (ThermoFisher Scientific, Waltham, MA, USA) and N-(3-Dimethylaminopropyl)-N'Ethylcarbodiimide hydrochloride (EDAC; Merck) at a final concentration of $0.1 \mathrm{M}$ in phosphate buffer saline (0.137 M NaCl; 0.0027 M KCl; 0.01 $\mathrm{M} \mathrm{Na}_{2} \mathrm{HPO}_{4} ; 0.0018$ $\mathrm{M} \mathrm{KH}_{2} \mathrm{PO}_{4}$ ), $150 \mathrm{mM} \mathrm{NaCl}$ and $3 \%$ sucrose. The mixture was incubated at $4^{\circ} \mathrm{C}$ for $18 \mathrm{~h}$, then dialyzed in a $3.5 \mathrm{kDa}$ cut-off membrane (SnakeSkin; ThermoFisher Scientific) to eliminate unbound biotin. The biotinylated OMVs (OMVB) $(13 \mathrm{mg})$ were mixed with the fusion protein of rhizavidin and SmTSP-2 (rRzvSmTSP-2; obtained as previously described in detail) ${ }^{26}$ at a ratio of 5:1 (mass/ mass) and incubated at $4^{\circ} \mathrm{C}$ for $18 \mathrm{~h}$.

\section{OMV:rSmTSP-2 Purification}

The OMV:rSmTSP-2 complex was purified by sizeexclusion chromatography in a Sephacryl S-200 XK 26/ 100 column (Cytiva, Marlborough, MA, USA), under pyrogen-free conditions. Blue Dextran 2000 (SigmaAldrich, Inc., St. Louis, MO, USA) was used to establish $\mathrm{V}_{0}$ (column void volume) of the column, peak $A$, and a protein with similar molecular weight (rRzvSmCD59, $26.9 \mathrm{kDa}$ ) was used to estimate the free rRzvSmTSP-2 protein elution volume. Aliquots corresponding to the $\mathrm{V}_{0}$ of the column and soluble protein were collected and analyzed by Western blot using an anti-serum previously generated in mice immunized with rSmTSP-2.

\section{Characterization of OMV:rSmTSP-2}

Particle size, polydispersity index (PDI), and zeta potential $(\zeta)$ were assessed by dynamic light scattering and electrophoretic mobility techniques using a Zetasizer Nano ZS90 (Malvern Panalytical Ltd., Malvern, UK). Aliquots (1 mL) of particle suspension were gently stirred and then analyzed at a fixed detection angle of $173^{\circ}$ and a temperature of $25^{\circ} \mathrm{C}$. The samples were measured with a Zetasizer version 7.12 software (Malvern); and results are presented as the mean $\pm \mathrm{SD}$ of three measurements.

\section{Transmission Electron Microscopy (TEM)}

Aliquots of purified, detoxified and biotinylated OMV (dOMV and OMV-B) and the OMV:rSmTSP-2 complex were fixed in Karnovsky's solution (4\% paraformaldehyde $+2 \%$ glutaraldehyde in $0.1 \mathrm{M}$ cacodylate buffer, $\mathrm{pH} 7$ ) for microscopy. A JEM-1230 transmission electron microscope (JEOL Ltd, Tokyo, Japan) was used for imaging and size confirmation. Aliquots $(20 \mu \mathrm{L})$ of the fixed samples of nanoparticle suspensions were dispersed in formvar film-coated grids and contrasted with $2 \%$ uranyl acetate.

\section{Mouse Dendritic Cell Generation and Activation}

C57BL/6 female mice of 4-5 weeks of age (supplied by the Breeding Facility of the Instituto Butantan) were euthanized in a $\mathrm{CO}_{2}$ chamber for femur removal. The bone marrow was flushed out with a syringe using RPMI-1640 medium (GIBCO by Life Technology, Paisley, UK) to obtain the bone marrow precursor cells. 
The cells were centrifuged at $11,000 \times \mathrm{g}$ for $10 \mathrm{~min}$ at $4^{\circ} \mathrm{C}$, and suspended in $10 \mathrm{~mL}$ RPMI-1640 medium supplemented with 5\% heat-inactivated fetal bovine serum (SigmaAldrich, Inc.), $0.5 \% \quad 10 \mathrm{mM} \quad \beta$-mercaptoethanol, $1 \%$ L-glutamine, 1\% MEM vitamins, $1 \%$ penicillin/ streptomycin and HEPES buffer (complete medium).

Cells were plated in 6 -well culture plates $\left(1 \times 10^{6}\right.$ cells/ $\mathrm{mL} ; 5 \mathrm{~mL} /$ well) in complete medium supplemented with $10 \mathrm{ng} / \mathrm{mL}$ GM-CSF and $5 \mathrm{ng} / \mathrm{mL}$ IL-4 (both from InVitrogen, ThermoFisher Scientific) at $37^{\circ} \mathrm{C}$ in $5 \% \mathrm{CO}_{2}$. On days 2 and 4 , the floating cells were gently removed, and fresh medium containing GM-CSF and IL-4 was added. On day 7, non-adherent cells and loosely adherent proliferating bone marrow-derived dendritic cell (BM-DC) aggregates were harvested, resuspended, counted, separated in microtubes containing $1 \times 10^{6}$ cells in $1 \mathrm{~mL}$ complete medium (modified from). ${ }^{27}$ The BM-DC were stimulated with LPS (10 ng/mL, from E. coli O55:B5; Sigma-Aldrich, Inc.), OMV-B (1 $\mu \mathrm{g} / \mathrm{mL})$, rRzv:SmTSP-2 $(1 \mu \mathrm{g} / \mathrm{mL})$ or OMV:rSmTSP-2 $(1 \mu \mathrm{g} / \mathrm{mL})$ and plated in $96-$ well plates $(0.25 \mathrm{~mL} /$ well in quadruplicate $)$ and incubated for $20 \mathrm{~h}$ at $37^{\circ} \mathrm{C}$ in $5 \% \mathrm{CO}_{2}$. The supernatant was recovered and stored at $-20^{\circ} \mathrm{C}$ for cytokine quantification.

\section{Analysis of Surface Markers and Cytokines by Flow Cytometry}

The analysis of surface markers was performed on mouse BM-DC after stimulation with antigens as described above. Stimulated cells were resuspended in flow cytometry staining buffer, pooled and incubated with anti-Fc $\gamma$ RII $/$ RIII receptor monoclonal antibodies $(\mathrm{mAb})$ for $15 \mathrm{~min} /$ $4^{\circ} \mathrm{C}$. Then, BM-DCs cells $\left(1 \times 10^{6}\right)$ were stained with antimouse anti-CD11c-PE-Cy7 (clone N418), anti-CD40BV421 (clone 3/23); anti-CD80-FITC (clone 16-10A1); anti-CD86-PE (clone GL-1) or anti-MHC-II-FITC (clone AF6-120.1) $\mathrm{mAbs}$ (BD Biosciences) for $30 \mathrm{~min}$ at $4^{\circ} \mathrm{C}$ in the dark. BM-DCs were incubated with the viability reagent (BD Biosciences) according to the manufacturer's protocol and fixed with $0.1 \%$ paraformaldehyde (SigmaAldrich, Inc., St. Louis, MO, USA) in PBS. The gating strategy is displayed in Supplementary Figure 1. The fluorescent intensities of the cells were measured by flow cytometry and the results expressed as Mean Fluorescence Intensity (MFI). Cell acquisition of at least 30,000 events per sample was performed in a FACS Canto II flow cytometer (BD Biosciences) and data were analyzed using the FlowJo 10 software.
The levels of proinflammatory cytokines (TNF- $\alpha$, IL-6 and IL-12) or IL-10 were measured after $24 \mathrm{~h}$ of stimulation with the different antigens from the BM-DCs supernatants using ELISA Kits (PeproTech, Cranbury, NJ, USA), according to the manufacturer's instructions.

\section{Mouse Immunization}

Groups of C57BL/6 female mice (4-5-week-old) were inoculated intraperitoneally with $500 \mu \mathrm{L}$ of either $5 \mu \mathrm{g}$ of rSmTSP-2, a mix of $2 \mu \mathrm{g}$ rSmTSP-2 and $8 \mu \mathrm{g}$ of dOMV or $10 \mu \mathrm{g}$ of the OMV:rSmTSP-2 complex, all with aluminum hydroxide, Alum, 1:9 (mass protein: mass Alum) (Brenntag Specialties, Inc., South Plainfield, NJ), or sterile saline (with the highest amount of Alum). Mice were immunized with three doses 15 days apart. All experiments were performed in triplicate.

\section{SmTSP-2 Specific Responses in Spleen Cells}

Fifteen days after each immunization, splenocytes were obtained from five animals in each group. Organs were dissociated with a Potter tissue homogenizer (Corning, Inc., Corning, NY, USA), and erythrocytes were lysed with Gey's solution $\left(0.15 \mathrm{M} \mathrm{NH} \mathrm{NH}_{4} \mathrm{Cl}\right.$ and $10 \mathrm{mM}$ $\left.\mathrm{KHCO}_{3}\right)$. The cells were plated in 24 -well plates $(1 \times$ $10^{6}$ cells $/ \mathrm{mL} ; 1 \mathrm{~mL}$ per well) and incubated either in RPMI medium $+10 \%$ FBS (non-stimulated), stimulated with rSmTSP-2 $(10 \mu \mathrm{g} / \mathrm{mL})$ or Concanavalin A $(10 \mu \mathrm{g} /$ $\mathrm{mL}$; Sigma-Aldrich, Inc.) as positive control, and incubated in a $5 \% \mathrm{CO}_{2}$ incubator for 24 at $37^{\circ} \mathrm{C}$. Culture supernatants were collected to evaluate cytokine production using the BD ${ }^{\text {TM }}$ Cytometric Bead Array - Th1/Th2/ Th17 kit (BD Biosciences), following the manufacturer's recommendations.

For intracellular cytokine detection, spleen cells were plated in 96-well plates $\left(1 \times 10^{6}\right.$ cells per well $)$, incubated either in RPMI medium $+10 \%$ FBS (non-stimulated), stimulated with rSmTSP-2 $(10 \mu \mathrm{g} / \mathrm{mL})$ or Concanavalin A $(10 \mu \mathrm{g} / \mathrm{mL})$ as positive control, and incubated in a $5 \%$ $\mathrm{CO}_{2}$ incubator for $4 \mathrm{~h}$ at $37^{\circ} \mathrm{C}$. Monensin solution $(3 \mu \mathrm{M})$ (eBioscience $^{\mathrm{TM}}$, ThermoFisher Scientific) was then added to the cells and incubated for a further $6 \mathrm{~h}$. The cells were harvested and labeled with PercP-anti-mouse CD4 (clone RM4-5) or PE-Cy7-anti-mouse CD8 (clone 53-6.7)(BD Biosciences) for $30 \mathrm{~min}$, then fixed and permeabilized with BD Cytofix/Cytoperm ${ }^{\mathrm{TM}}$ solution kit (BD Biosciences), according to the manufacturer's instructions, 
and further incubated with FITC-anti-mouse TNF- $\alpha$ (clone MP6-XT22), PE-anti-mouse IL-4 (clone 11B11), APC-anti - mouse IFN- $\gamma$ (clone XMG1.2) and BV421-anti-mouse IL-2 (clone JES6-5H4) (all BD Biosciences) for $30 \mathrm{~min}$. Cell acquisition of at least 100,000 events per sample was performed in a FACS Canto II flow cytometer (BD Biosciences) and data were analyzed using the FlowJo 10 software. The results are displayed as the number of each cell type in the total of spleen cells obtained.

For evaluation of the number and activation of neutrophils, eosinophils and macrophages, the splenocytes were plated in 96-well plates $\left(1 \times 10^{6}\right.$ cells per well $)$ and treated as described above. The cells were then labeled with BB515-anti-mouse CD11b (clone M1/70), PE.Cy7-antimouse CD11c (clone HL3), APC-anti-mouse Ly6G (clone 1A8), BV421-anti-mouse F4/80 (clone T45-2342) (all BD Biosciences) for $30 \mathrm{~min}$. Then, the cells were fixed and permeabilized with BD Cytofix/Cytoperm Kit and further incubated with PE-anti-mouse IL-4 (clone 11B11) for $30 \mathrm{~min}$. Cell acquisition of at least 100,000 events per sample was performed in a FACS Canto II flow cytometer, and data were analyzed using the FlowJo 10 software. $^{28}$ The results are displayed as the total number obtained of each IL-4 producing cell type.

\section{Enzyme-Linked Immunosorbent Assay (ELISA)}

Fifteen days after each immunization (before each new dose), blood samples were collected from the immunized mice. Serum was separated by centrifugation and stored at $-20^{\circ} \mathrm{C}$. Ninety-six well polystyrene plates $\left(\mathrm{Nunc}^{\circledR}\right.$, ThermoFisher Scientific) were coated with rSmTSP-2 (100 $\mu \mathrm{L}$ of a $10 \mu \mathrm{g} / \mathrm{mL}$ rSmTSP-2 solution per well) in $0.05 \mathrm{M}$ sodium carbonate/bicarbonate buffer $(\mathrm{pH} 9.8)$ and incubated at $4{ }^{\circ} \mathrm{C}$ overnight. The wells were blocked for 30 min with PBS containing 5\% skim milk. The serum samples were serially diluted starting at 1:400, added to the wells and incubated for $2 \mathrm{~h}$ at $37^{\circ} \mathrm{C}$. Biotinylated secondary antibodies (anti-mouse IgG, IgG1 or IgG2c; PharMingen $^{\circledR}$, BD Biosciences) diluted 1:5000 were added to the wells, which were then incubated for $2 \mathrm{~h}$ at $37^{\circ} \mathrm{C}$. Streptavidin peroxidase was added in 1:1000 dilution and the plates were incubated again for $1 \mathrm{~h}$ at $37^{\circ} \mathrm{C}$. Substrate solution $(100 \mu \mathrm{L}$ of $0.5 \mathrm{mg} / \mathrm{mL}$ o-phenylenediamine, Sigma Aldrich, Inc., in 0.1 M sodium citrate buffer, $\mathrm{pH} 5$ ) was added and the reaction maintained at room temperature to develop. The reaction was interrupted by the addition of $\mathrm{H}_{2} \mathrm{SO}_{4}$, and the absorbance at $492 \mathrm{~nm}$ was read with an ELISA reader (Epoch Microplate Spectrophotometer; BioTek Instruments, Winooski, VT, USA). The results are presented as antibody titers (the inverse of the highest dilution giving positive results) or in arbitrary units (AU) vs a standard curve using dilutions of an anti-SmTSP-2 hyperimmune sera. One arbitrary unit (1 AU) is equivalent to the reciprocal plasma dilution that gives an OD of 1.0 over background.

\section{Statistical Analysis}

The statistical significance of differences between experimental groups was analyzed using one-way analysis of variance (ANOVA) followed by Tukey's multiplecomparisons test (GraphPad Prism 5.0; GraphPad Software, Inc.); $p<0.05$ was considered statistically significant. Values are reported as the mean $\pm \mathrm{SD}$.

\section{Results \\ Construction of OMV-B:rRzvSmTSP-2 Nanoparticles}

The fusion protein rRzvSmTSP-2 was expressed in E. coli, purified and detoxified as previously described. $^{26}$ OMVs were isolated from $N$. lactamica and detoxified using DOC treatment. Detoxified OMVs were chemically conjugated to the biotin derivative Amine-PEG3-Biotin, to provide a coating of binding sites for the rhizavidin-displaying antigen. Once combined, the fusion antigen spontaneously attaches to the biotinylated OMVs via the high-affinity binding between biotin and rhizavidin, resulting in an OMV:rSmTSP-2 macromolecular complex exposing the SmTSP-2 antigen on its surface (Figure 1).

\section{OMV:rSmTSP-2 Nanoparticle Purification}

The assembled OMV:rSmTSP-2 nanoparticles were isolated by size-exclusion chromatography in the early elution fractions, due to their high molecular weight (Figure 2A, peak A, green line), whereas the unbound antigen would separate in latter fractions (Figure 2A, peak B, red line). However, no protein was detected in peak B fractions (Figure 2A, green line), indicating that most of the recombinant protein was bound to the OMVs. The rRzvSmTSP-2 protein was visualized by Western blot in peak A fractions of the elution (Figure 2B), confirming its association to biotinylated OMVs. 
A

ss

HHHHHH

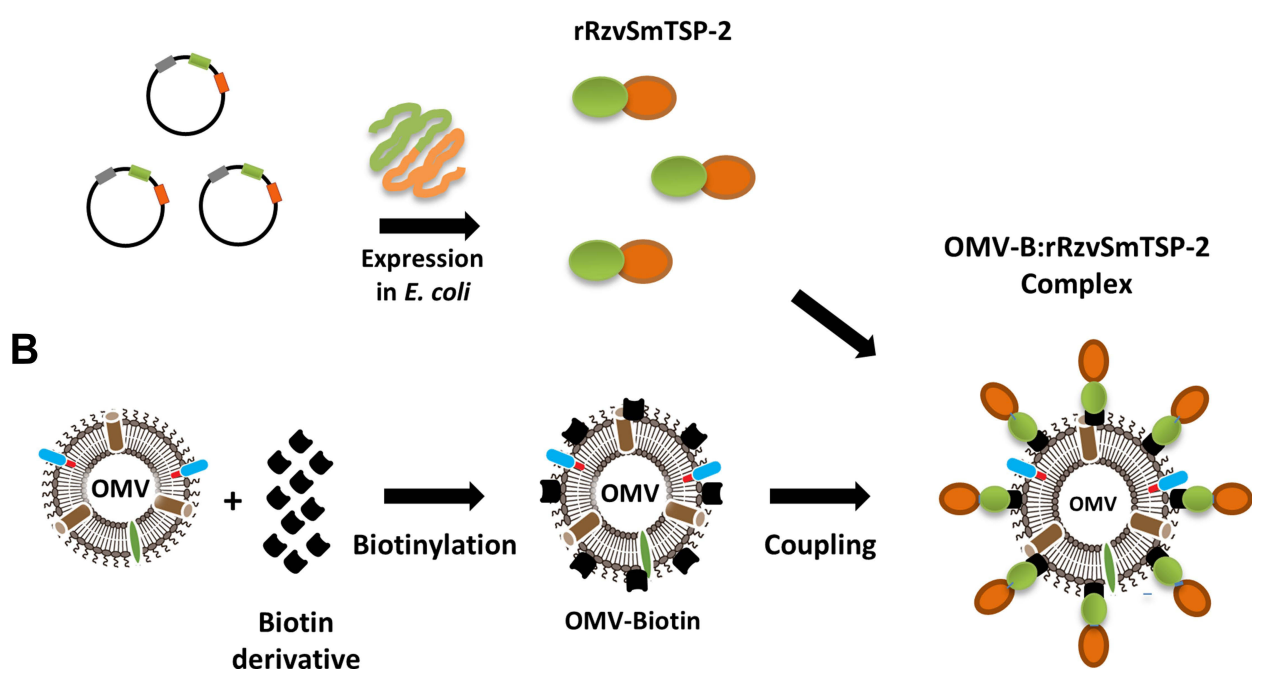

Figure I Schematic representation of the assembly of the OMV:rSmTSP-2 complex. (A) Representation of the expression cassette containing the antigen gene in fusion with the rhizavidin (rzv) gene. A secretion signal sequence is inserted in the $\mathrm{N}$-terminus and a Histidine tag in the C-terminus of the sequence. ${ }^{22}$ Representation of $E$. coli expression of the rRzvTSP-2 protein. (B) Biotinylation of OMV and coupling with the fusion protein to produce the OMV:rSmTSP-2 multimolecular complex.

Abbreviations: rzv, rhizavidin; ss, secretion signal sequence; $\mathrm{HHHHHH,} \mathrm{6xHis} \mathrm{tag,} \mathrm{OMV,} \mathrm{outer} \mathrm{membrane} \mathrm{vesicles.}$

A



B

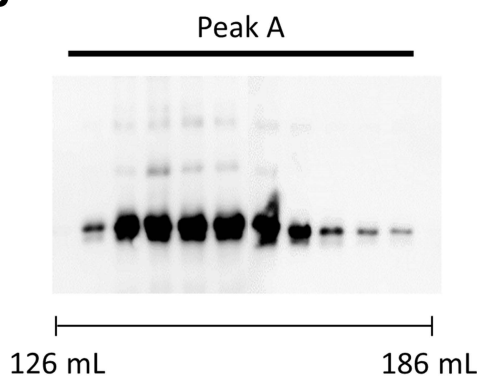

Figure 2 Purification of the OMV:rSmTSP-2 complex. (A) Gel filtration chromatography fractions by absorbance at $280 \mathrm{~nm}$. Peak $\mathrm{A}$ is the $\mathrm{V}_{0}$ of the column determined by Blue Dextran 2000 (blue line); peak B is free protein not bound to OMVs (red line); Sample fractions obtained in the purification (green line). (B) Western blot of the fractions that compose peak A visualized with anti-rSmTSP-2 antibody.

\section{OMV:rSmTSP-2 Biophysical Characterization}

Electron microscopy of the purified, detoxified, biotinylated OMVs and the OMV:rSmTSP-2 complex showed that the vesicles remained spherical after the sequential procedures for endotoxin removal, biotinylation and coupling with the antigen (Figure 3A-C). The electrophoretic profiles of the OMVs, rSmTSP-2 and OMV:rSmTSP-2 complexes showed the presence of the band corresponding to rSmTSP-2 and another one corresponding to PorB, the major protein present on the surface of the $N$. lactamica OMVs (Figure 3D). Particle size analysis showed that purified OMVs and dOMV are homogeneously distributed, presenting moderate polydispersity (PDI 0.21 and 0.20 ) and a mean hydrodynamic size of 80.1 and $178.5 \mathrm{~nm}$, respectively. Biotinylated OMVs and the OMV:rSmTSP2 complexes display a higher polydispersity (PDI 1.0 and 0.49) and a mean hydrodynamic size of 399.9 and 196.3 $\mathrm{nm}$, respectively (Table 1). After antigen coupling, the $\zeta$ potential of the complex becomes more negative.

Evaluation of the endotoxin activity indicated that the detoxification process was effective in decreasing by 100 times the amount of active endotoxin on the OMV membrane. Purification steps following biotinylation and antigen coupling further decreased the LPS activity, with the OMV complexes being within the accepted endotoxin 


\section{Purified OMVs}

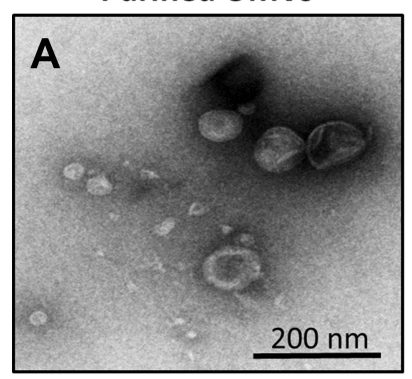

D

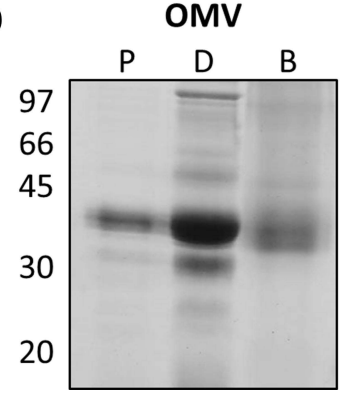

Det-OMVs



rRzvSmTSP-2

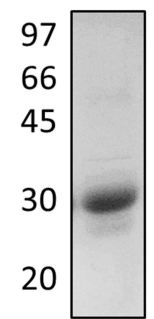

OMV:rSmTSP-2

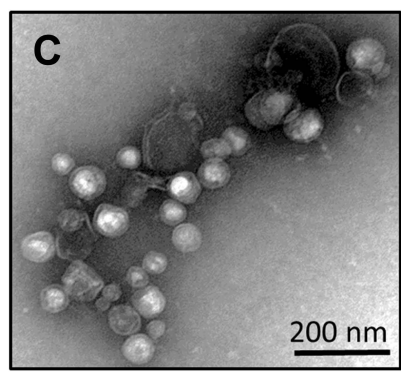

OMV:rSmTSP-2



Figure 3 Biophysical characterization of the OMV:rSmTSP-2 complex. Transmission electron microscopy (TEM) of (A) purified OMVs isolated from N. lactamica; (B) dOMVs; (C) the OMV:rSmTSP-2 complex. (D) Electrophoretic profile of purified (P), detoxified (D) and biotinylated (B) OMVs; purified rRzvSmTSP-2 and the purified OMV:rSmTSP-2 complex.

range for OMV-containing products $(<400 \quad \mathrm{EU} / \mu \mathrm{g})$ (Table 1). ${ }^{29}$

\section{Activation of Dendritic Cells by}

\section{S. mansoni Antigen Formulations}

In order to investigate possible induction of innate immune responses, mouse BM-DCs were exposed to the rSmTSP-2 soluble antigen or to the OMV:rSmTSP-2 complex, and the expression of co-stimulatory markers and production of immunomodulatory cytokines were assessed. In the case of surface molecules involved in antigen presentation, the expression of CD40 and CD86 was increased by LPS and OMVs (both nonconjugated and conjugated with antigen) but not by the soluble antigen, while the expression of CD80 was not substantially affected by any of the agents (Figure 4A) (FACS display in Supplementary Figure 1). The results show that the production of TNF- $\alpha$ and IL-12 were induced equally well by the soluble and conjugated antigen, at levels comparable to LPS (used as positive control) or dOMVs (Figure 4B). Conversely, the production of IL-6 was induced by LPS and the OMV nanoparticles more abundantly than by the soluble antigen, as did the production of IL-10 (Figure 4B). On the other hand, similar to TNF- $\alpha$, MHC-II expression was increased to a different degree by all agents (Figure 4A). Thus, IL-12 production in mouse DCs seems to be stimulated by the soluble antigen rather than by the nanoparticles, while both are active in inducing TNF- $\alpha$ and IL-6 production and increasing MHC-II expression; only the nanoparticles/LPS can induce increased CD40 and CD86 expression and IL-10 production.

\section{OMV:rSmTSP-2 Particles Induce Antigen-Specific Cellular Immune Responses}

To evaluate the induction of cellular immune responses, groups of mice were immunized with three doses of either

Table I OMV Physical Characteristics and Endotoxin Content

\begin{tabular}{|l|c|c|c|c|}
\hline Nanoparticles & Hydrodynamic Size (nm) \pm SD & Polydispersion Index (PDI) & $\zeta$ Potential (mV) \pm SD & Endotoxin (EU/mg)* \\
\hline Purified OMV & $80.1 \pm 4.21$ & 0.210 & $-10.1 \pm 0.19$ & $15,600,000-156,000,000$ \\
Detoxified OMV & $178.5 \pm 1.65$ & 0.203 & $-8.09 \pm 0.23$ & $1,560,000-15,600,000$ \\
Biotinylated OMV & $399.9 \pm 4.22$ & 1.000 & $-8.04 \pm 0.54$ & $31,200-312,000$ \\
OMV:rSmTSP-2 & $196.3 \pm 6.73$ & 0.497 & $-28.03 \pm 6.07$ & $83,000-830,000$ \\
\hline
\end{tabular}

Note: *Endotoxin activity determined by gel clot LAL assay. 
A
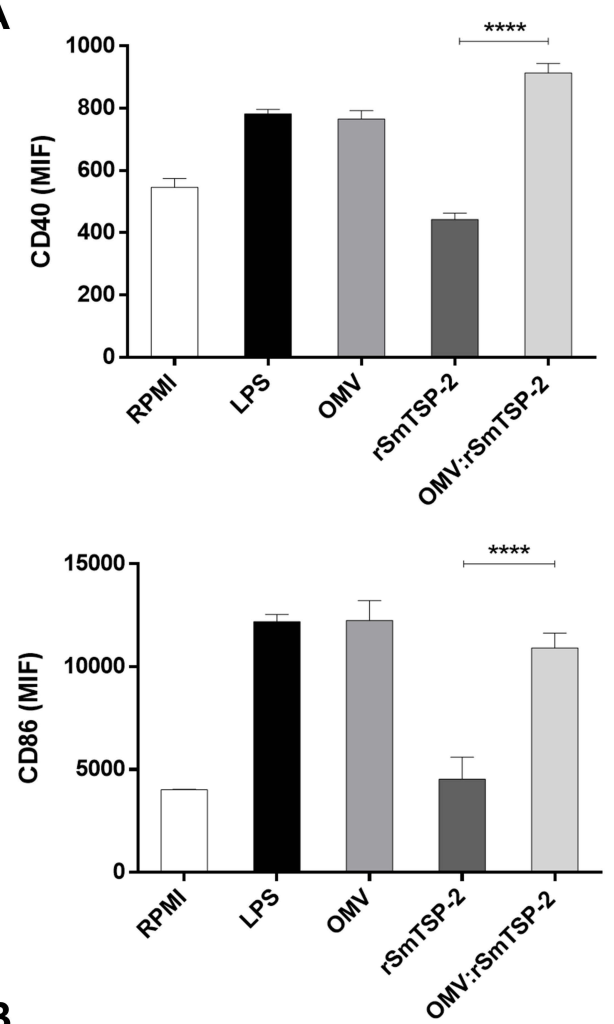

$\mathbf{B}$
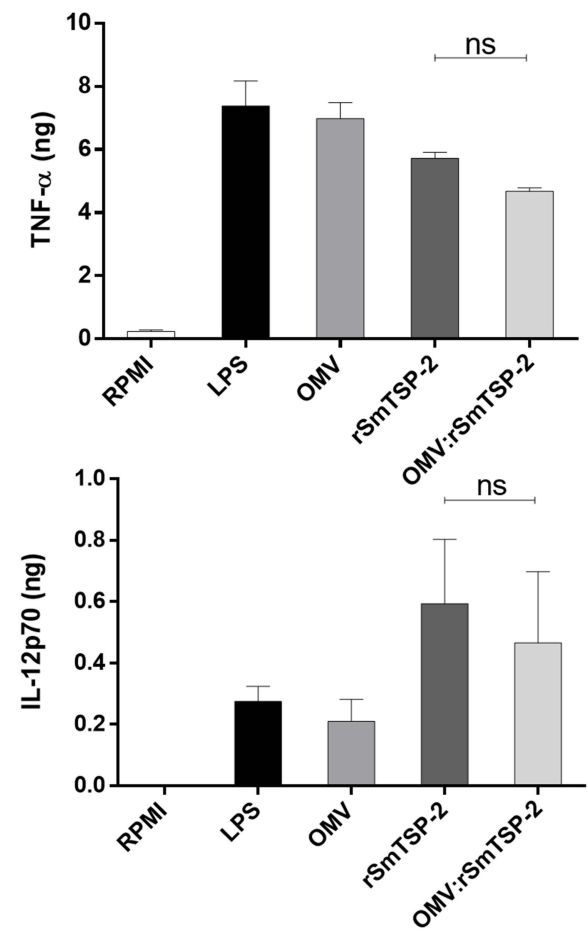
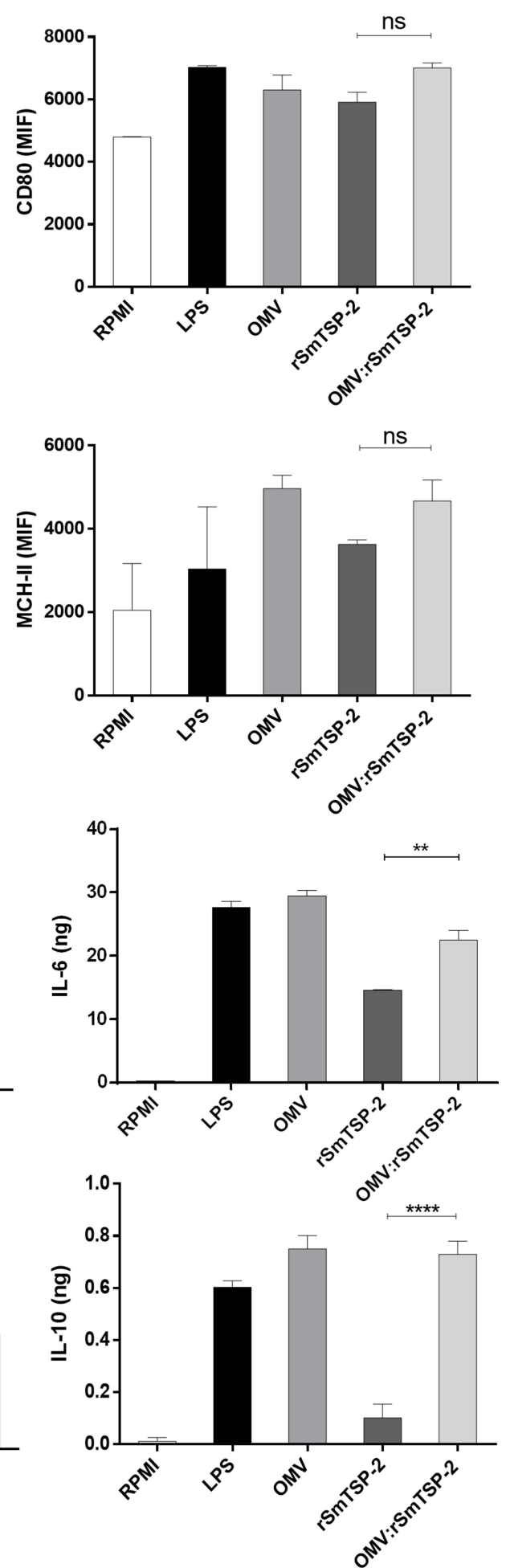

Figure 4 Activation of mouse dendritic cells by the OMV:rSmTSP-2 complex. Expression of co-stimulatory markers and production of immunomodulatory cytokines by mouse BM-DC was measured after exposure for $24 \mathrm{~h}$ to either culture medium alone (RPMI, negative control), LPS (I0 ng/mL; positive control), OMV-B (I $\mu \mathrm{g} / \mathrm{mL}), \mathrm{rRzv}$ : SmTSP-2 (I $\mu \mathrm{g} / \mathrm{mL})$ or the OMV:rSmTSP-2 complex (I $\mu \mathrm{g} / \mathrm{mL})$. The response was measured as (A) expression of the surface molecules CD40, CD80, CD86 and MHC-II; (B) production of TNF- $\alpha$, IL-6, IL-12 and IL-10. Data are reported as mean \pm SD of 2-4 replicate samples and expressed in MFI (mean fluorescence intensity) for surface marker molecules and $\mathrm{ng} / 10^{6}$ cells for cytokine production. Statistical analysis was performed with One-way ANOVA. **p $<0.01$; $* * * * p<0.000 \mathrm{I}$.

Abbreviation: ns, not significant. 
the rSmTSP-2 antigen, a mixture of rSmTSP-2 and dOMV, or the OMV:rSmTSP-2 nanoparticles. The antigen-specific cell-mediated immune response was assessed in vitro by exposing splenocytes from immunized mice to the cognate antigen rSmTSP-2. Splenocytes were collected 15 days after the second or the third vaccine dose, and cellular immunity was evaluated as cytokine production in the culture supernatant. After 2 doses, production of IL-6, IL-17 and IL-10 was higher in the group receiving OMV: rSmTSP-2 as compared to the groups immunized with rSm-TSP-2 alone or the combination of antigen and dOMV (Figure 5). After the third dose, splenocytes from both the mixture and the OMV:rSmTSP-2 immunized groups produced higher levels of these cytokines (Figure 5). No difference between groups was identified in terms of production of IFN- $\gamma$, TNF- $\alpha$, IL-4 and IL-2.

In parallel, we investigated whether immunization with the nanoparticle complex could drive a distinct lymphocyte phenotype in comparison to the administration of the recombinant antigen. Splenocytes from immunized animals were stimulated with rSmTSP-2 in vitro and analyzed by flow cytometry. $\mathrm{CD} 4^{+}$and $\mathrm{CD} 8^{+} \mathrm{T}$ cells expressing TNF- $\alpha$, IFN- $\gamma$, IL- 4 and IL-2 were determined using specific antibodies. Mice immunized with OMV:rSmTSP-2 showed an increased number of $\mathrm{CD}^{+}$and $\mathrm{CD} 8^{+} \mathrm{T}$ cells expressing TNF- $\alpha$ and IL-4 (Figure 6) as compared to the rSmTSP-2 group. Moreover, the OMV:rSmTSP-2 group showed a significantly higher number of $\mathrm{CD}^{+} \mathrm{T}$ cells expressing IFN- $\gamma$ and IL-2 as compared to rSmTSP-2 immunized animals (Figure 6).

The flow cytometry experiments had indicated that production of IL-4 could derive from activation of other immune cells, such as macrophages, neutrophils and eosinophils. Splenocytes of mice immunized with either the rSmTSP-2 protein or the OMV:rSmTSP-2 complex were stimulated with rSmTSP-2 and stained for the different cell types and IL-4. The OMV:rSmTSP-2 group showed increased IL-4 production in macrophages $(\mathrm{F} 4 / 80+\mathrm{CD} 11 \mathrm{c}$ $+)$ and neutrophils (Ly6G+CD11b+) as compared to the rSmTSP-2 immunized group (Figure 7). There is no difference in IL-4 production in eosinophils (Ly6G+F480+) between the groups.

\section{OMV:rSmTSP-2 Particles Induce Antigen-Specific Antibody Responses}

Immunization of mice with rSmTSP-2 induced a significant anti-SmTSP-2 IgG antibody response
(Figure 8A). Total anti-SmTSP-2 IgG titers in mice immunized with the OMV:rSmTSP-2 complex was 100-fold higher than that observed in mice that received the rSmTSP-2 protein (Figure 8A). Immunization with rSmTSP-2 induced mainly antibodies of the IgG1 isotype, while immunization with the OMV:rSmTSP-2 nanoparticles induced a more balanced profile, displaying both IgG1 and $\operatorname{IgG} 2 \mathrm{c}$, and at much higher levels (Figure 8B). Additionally, we measured antibody levels as arbitrary units against a standard curve of specific hyperimmune sera. The nanoparticle complex induced an increase of $\sim 10$ $\log _{2}$ AU of specific anti-rSmTSP-2 antibodies (Supplementary Figure 2).

\section{Discussion}

Live attenuated Schistosoma cercariae are a highly effective vaccine model in eliciting broad and long-lasting immunity and protection against challenge infection with cercariae. $^{30}$ However, the use of attenuated parasites would pose important safety concerns and, additionally, significant difficulties in a production process in compliance with good manufacturing practice. These limitations encourage the development of subunit vaccines ${ }^{31}$ or vaccines that include defined antigens and components. The poor immunogenicity of recombinant proteins often leads to the addition of adjuvants or to the development of new antigen-presenting systems. ${ }^{32-34}$

OMVs have several advantages in optimizing antigen presentation, eg, the trace amounts of LPS in the membranes have significant adjuvant properties with reduced toxicity, and the nanoparticle structure engage antigenpresenting cells in particular, but also non-specific amplification of the immune response, such as innate immunity. ${ }^{16,35}$ Genetically engineered OMVs have been successful in antigen presentation. ${ }^{33}$ The fusion of different antigens with cytolysin A (ClyA) was shown to be translocated to $E$. coli-derived OMVs displaying the antigens on the vesicle surface, ${ }^{36}$ but the type of protein may interfere with externalization of the fusion protein. ${ }^{37}$ Therefore, there are challenges in the use of these recombinant vehicles and alternative strategies for effective coupling to OMV surfaces are pursued. The MAPS technology was first described for efficient coupling of recombinant proteins to polysaccharides. ${ }^{22}$ Furthermore, it was shown that presentation of antigens in larger complexes (of biotinylated dextran) induced significantly higher immune responses as compared to antigens in smaller complexes. ${ }^{22}$ In this study, we describe the use 
$2^{\text {nd }}$ Dose
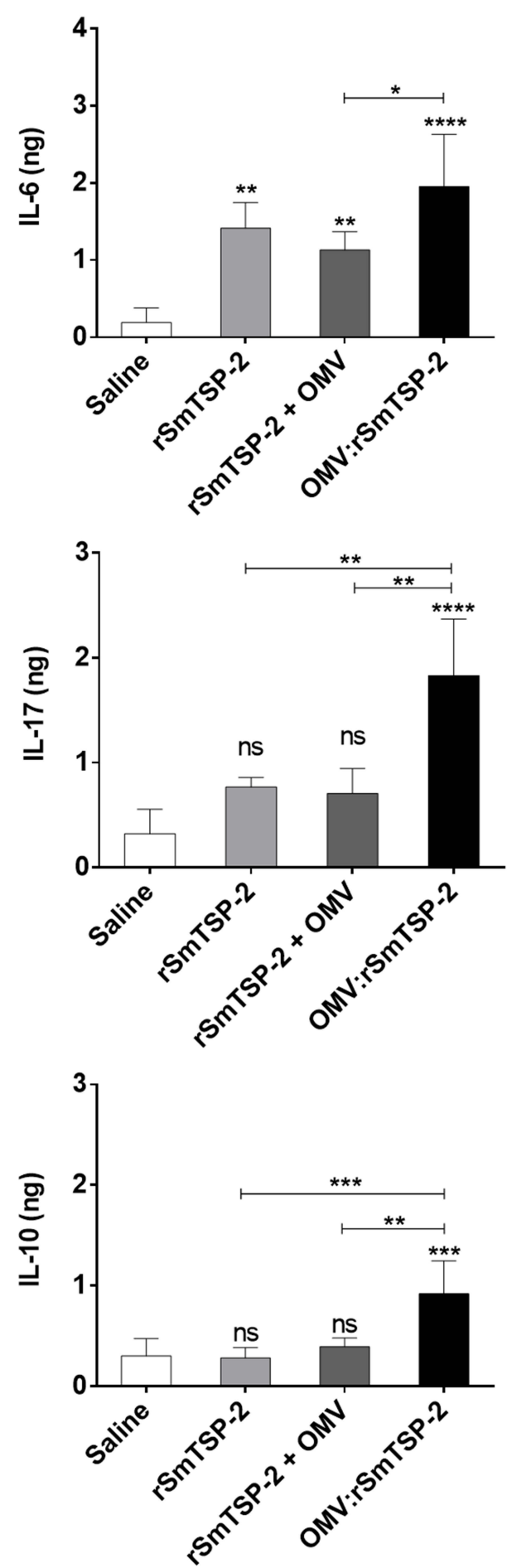

$3^{\text {rd }}$ Dose
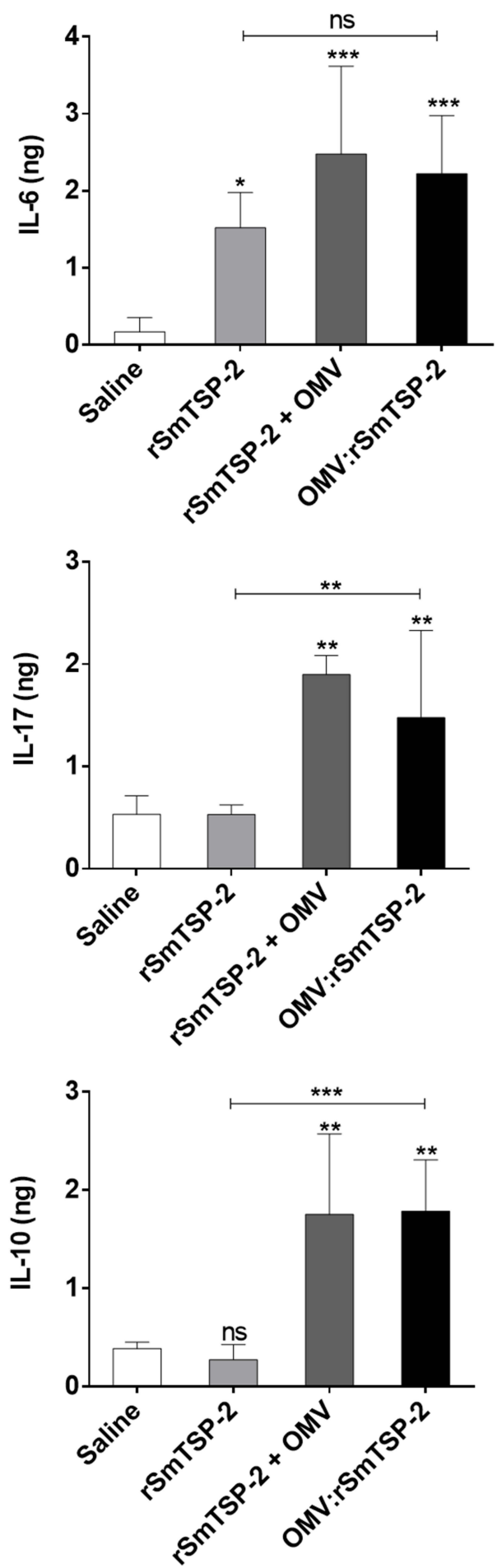

Figure 5 Antigen-specific cytokine production by splenocytes of immunized mice. Mice received either rSmTSP-2 (5 $\mu \mathrm{g}), \mathrm{dOMVs}(10 \mu \mathrm{g}), \mathrm{rSmTSP}-2(2 \mu \mathrm{g})+\mathrm{dOMVs}(8 \mu \mathrm{g})$ or OMV:rSmTSP-2 (10 $\mu \mathrm{g}$ ), all with Alum, 1:9 (mass protein: mass Alum); or Saline (with the highest amount of Alum) in 3 doses with I5 days interval. Splenocytes were recovered after the 2 nd and the 3 rd dose and stimulated in vitro for $24 \mathrm{~h}$ with the rSmTSP-2 protein. Cytokine production was analyzed by Cytometric Bead Array in the cell culture supernatant. The results are reported as the mean \pm SD of values (ng/l $0^{6}$ cells) from 5 animals. Statistical significance between groups was determined by Oneway ANOVA. *p $<0.1$; **p $<0.01$; ***p $<0.001$; ****p $<0.0001$.

Abbreviation: ns, not significant. 
$\mathrm{CD}^{+} \mathrm{T}$ cell
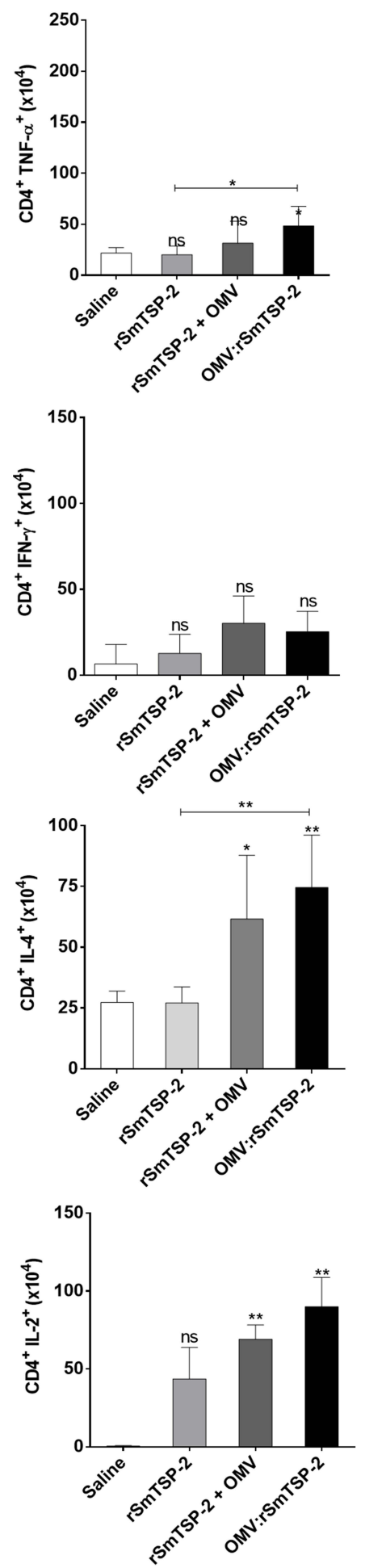

$\mathrm{CD}^{+} \mathrm{T}$ cell
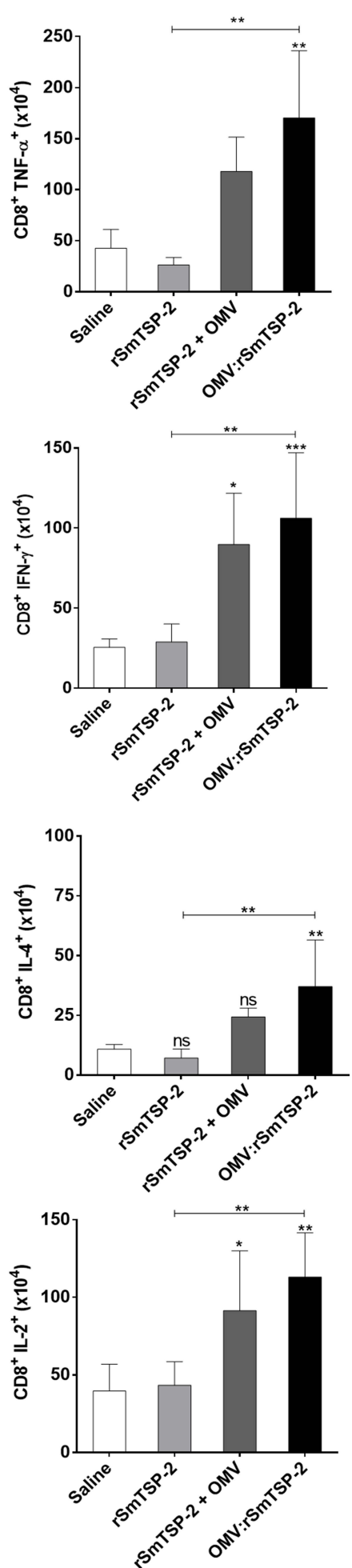

Figure 6 Antigen-specific cytokine production by $\mathrm{CD} 4^{+}$and $\mathrm{CD} 8^{+} \mathrm{T}$ cells from immunized mice. Splenocytes recovered from mice that received either, $\mathrm{rSmTSP}-2(5 \mu \mathrm{g})$, dOMVs (10 $\mu \mathrm{g})$, rSmTSP-2 $(2 \mu \mathrm{g})+\operatorname{dOMV}(8 \mu \mathrm{g})$ or OMV:rSmTSP-2 $(10 \mu \mathrm{g})$, all with Alum, I:9 (mass protein: mass Alum); or Saline (with the highest amount of Alum) in 3 doses with 15 days interval were stimulated in vitro with rSmTSP-2, then labeled with anti-CD3, anti-CD4 and anti-CD8 antibodies (for immunophenotyping) and with antibodies to TNF- $\alpha$, IFN- $\gamma$, IL-4 and IL-2 (to detect the intracellular cytokine production) and analyzed by flow cytometry. The results are reported as the mean \pm SD of values (in $10^{6}$ cells) from 5 animals. Statistical analysis was performed by One-way ANOVA; differences vs the saline group or between the different groups as indicated by bars. $* p<0.1 ; * * p<0.01 ; * * * 00.001$.

Abbreviation: ns, not significant. 
A



B

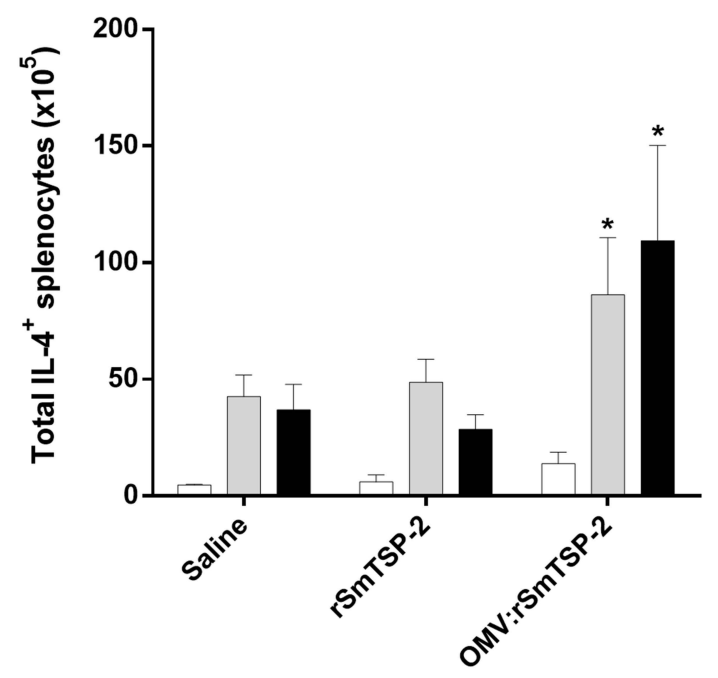

Eosinophil $\square$ Macrophage $\square$ Neutrophil

Figure 7 IL-4 production by eosinophils, macrophages and neutrophils from immunized mice. Splenocytes of mice immunized with 3 doses of either rSmTSP-2 $(2 \mu g)$ or OMV:rSmTSP-2 $(10 \mu \mathrm{g})$, all with Alum, 1:9 (mass protein: mass Alum); or Saline (with the highest amount of Alum) were analyzed by flow cytometry as to immune cell populations. (A) Total number of splenocytes: eosinophils $\left(\right.$ Ly $\left.6 \mathrm{G}^{+} \mathrm{F} 480^{+}\right)$, macrophages $\left(\mathrm{F} 4 / 80^{+} \mathrm{CDIIc}{ }^{+}\right)$, and neutrophils $\left(\mathrm{Ly} 6 \mathrm{G}^{+} \mathrm{CDIIb}{ }^{+}\right)$. (B) Total IL-4+ producing eosinophils, macrophages and neutrophils in splenocytes from immunized mice. The results are reported as the mean \pm SD of values from 5 animals. ${ }^{*} p<0.05$.

of the OMV as scaffold, decorating its surface with the antigens using the MAPS technology. This approach combines the adjuvant properties of OMV with an abundant presence of specific antigens on the OMV surface, properties that are expected to enhance immunogenicity.

Characterization of the OMV:rSmTSP-2 nanoparticles by light scattering revealed that throughout the process there was an increase in the average size and in polydispersion, according to the intensity-weighted mean hydrodynamic size data. The $\zeta$ potential of the intermediate OMV preparations was slightly negative during the production process, and increased significantly after rSmTSP-2 binding, probably due to the negative charge of the rRzvSmTSP-2 fusion protein, pI 6.78 (https://web. expasy.org/compute_pi/). Colloids with higher $\zeta$ potential are more stable, while a low zeta potential is related with flocculation or coagulation. ${ }^{38}$

Effective antigen presentation includes the activation of antigen-presenting cells (APC), in particular (in the case of a primary immune response as in vaccination) dendritic cells, which are the APC mostly involved in the presentation of antigens to naïve $\mathrm{T}$ cells in lymph nodes. ${ }^{39,40}$ To examine the capacity of the OMV:rSmTSP-2 complex to activate DCs, we have exposed mouse BM-DCs to stimuli in vitro. DCs were activated by both soluble rSmTSP-2 and the OMV:rSmTSP-2 complex, but with notable differences. Both bare OMVs and the OMV:rSmTSP-2 complex induced upregulation of the co-stimulatory molecules CD40 and CD86, while the soluble antigen did not, an effect that may partly depend on the LPS displayed on the particle surface (as the same effect was observed with isolated LPS). Likewise, both particles upregulated the production of the multi-functional cytokine IL-6, together with the anti-inflammatory cytokine IL-10, while the soluble antigen was less efficient.

Conversely, the expression of MHC-II and the inflammatory cytokine TNF- $\alpha$ increased in response to both soluble rSmTSP-2 and OMV:rSmTSP-2. Furthermore, the soluble antigen significantly increased the production of the Th1/inflammatory cytokine IL-12, to the same level as the OMV-conjugated antigen but much higher than OMV, suggesting that IL-12 is induced by the antigens rather than the vesicles. Previous studies have shown that mice immunized with a chimeric rSmTSP-2 protein produced increased amounts of the IL-4, IL-10 and IFN- $\gamma$ cytokines. ${ }^{41}$ Overall, this shows that DCs are sensitive to LPS and to the OMV nanoparticles (with or without antigen) mostly in terms of immune regulation (upregulation of co-stimulatory molecules, production of IL-6 and IL10), while one of the key immunostimulatory Th1 cytokines (IL-12) is mainly triggered by the antigen (in soluble or particulate form). The components of OMVs encompass glycerophospholipids, lipopolysaccharide (LPS) and proteins, including periplasmic proteins. ${ }^{16,35}$ Therefore, OMVs do have antigenic components, which however do not seem to contribute to DC activation, besides the nonspecific LPS-dependent activation. It is notable that both particles and soluble antigen are effective in inducing/ upregulating two important players in antigen 
A

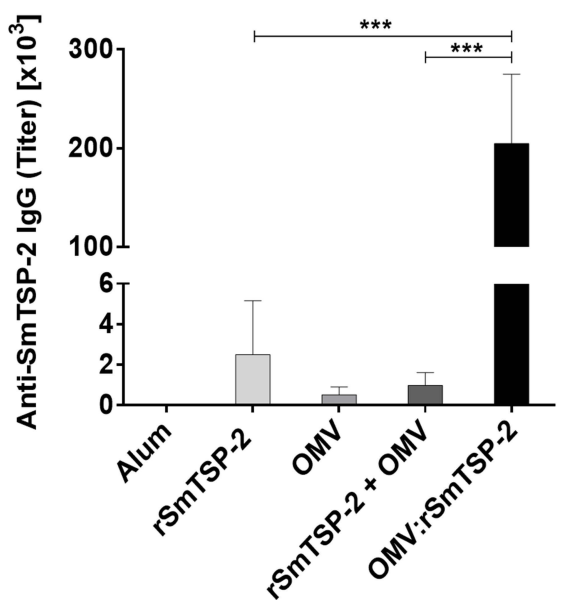

B

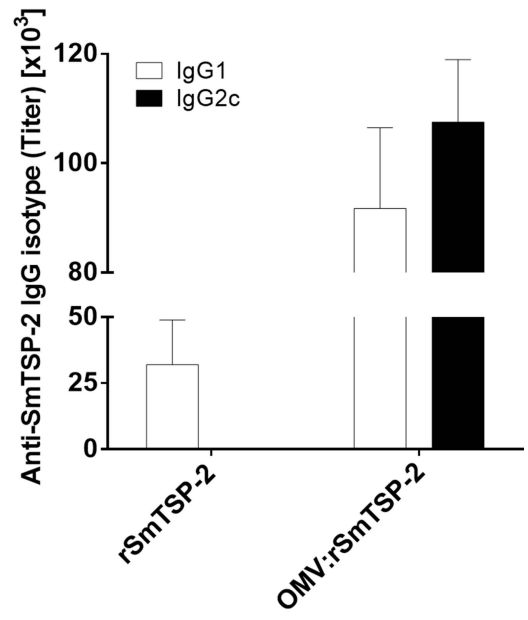

Figure 8 Specific antibody response against the rSmTSP-2 protein in immunized mice. (A) Total anti-rSmTSP-2 lgG antibodies in mice immunized with either, rSmTSP-2 (5 $\mu \mathrm{g})$, dOMVs $(10 \mu \mathrm{g}), \mathrm{rSmTSP}-2(2 \mu \mathrm{g})+\mathrm{dOMVs}(8 \mu \mathrm{g})$, or OMV:rSmTSP-2 (10 $\mu \mathrm{g})$, all with Alum, I:9 (mass protein: mass Alum); or Saline (with the highest amount of Alum) in 3 doses with 15 days interval. (B) $\operatorname{lgGI}$ and $\operatorname{lgG} 2 \mathrm{c}$ isotypes in groups immunized with rSmTSP-2 or OMV:rSmTSP-2. The results are reported as the mean titer \pm SD of values from 5 animals. Statistical analysis was performed with Two-way ANOVA; ***p $<0.00$ I.

presentation, ie, TNF- $\alpha$ and MHC-II. Thus, the profile of DC activation in response to soluble antigen includes increased TNF- $\alpha$, IL-12, IL-6 and MHC-II, while the profile upon exposure to the particulate antigens encompasses a wider activation spectrum that also include anti-inflammatory factors (increase in TNF- $\alpha$, IL-12, IL10, IL-6, CD40, CD86 and MHC-II), exploiting the activation capacity of OMV/LPS.

Mice immunized with the OMV:rSmTSP-2 complex developed antigen-specific cellular immunity, as assessed upon exposure of spleen cells from immunized mice to rSmTSP-2 in vitro. Stimulated splenocytes from OMV: rSmTSP-2 immunized mice show early production of IL6, IL-10 and IL-17 (after the second dose). After the third dose, spleen cells from mice immunized with either the OMV-rSmTSP-2 complex or the mixture of the antigen with OMV show increased levels of these cytokines. Increased IL-6 indicates the early induction of an inflammatory response, which can be regulated by IL- $10{ }^{42}$ IL17, an evolutionarily conserved immune defensive factor, plays an important role in defense against fungal, parasitic, bacterial and viral infections, mainly based on the induction of an inflammatory response aiming at eliminating the pathogen. ${ }^{43-45}$ Induction of an IL-17-dependent Th17 immune response is expected to contribute to protection against Schistosoma. ${ }^{46}$ It should be noted that immunization with the soluble antigen, despite the presence of Alum as adjuvant, resulted only in the antigen-specific induction of IL-6 production, with no detectable induction of IL-17 and IL-10, suggesting a much more limited immune activation.

Furthermore, the increased inflammatory response is confirmed by the finding that mice immunized with the OMV: rSmTSP-2 complex display an increased number of $\mathrm{CD}^{+}$ T cells producing TNF- $\alpha$ and IL- 2 in response to an antigen challenge, and of $\mathrm{CD}^{+} \mathrm{T}$ cells producing TNF- $\alpha$, IL- 2 and IFN- $\gamma$. Importantly, $\mathrm{CD}^{+} \mathrm{IFN}^{-} \gamma^{+} \mathrm{T}$ cells have been associated with the development of memory $\mathrm{CD}^{+} \mathrm{T}$ cells and with resistance to intracellular pathogens. ${ }^{47,48}$ Some antigens that are not accessible to antibodies can be detected by CD8 T cells, offering a broader range of protection. ${ }^{49}$ IFN- $\gamma$ also plays a crucial role in activation of Th1-mediated inflammatory adaptive immune responses. ${ }^{50}$

Notably, immunization with OMV:rSmTSP-2 induced an increase in the number of antigen-specific $\mathrm{CD} 4^{+} \mathrm{IL}-4^{+}$ $\mathrm{T}$ cells in the spleen, supporting the hypothesis of a concomitant activation of $\mathrm{Th} 2$ immune responses, which are associated with resistance to parasitic infections. $^{42,51}$ Furthermore, immunization with OMV: rSmTSP-2 also induced an increase in the number of IL4-producing macrophages and neutrophils in the spleen, and an increase in the number of eosinophils. IL-4 is a central cytokine in the type 2 immune response, involved in the activation of M2 macrophages and the production of $\mathrm{IgE}$ antibodies, important in the immune response against helminth infections. ${ }^{52}$ In helminth infections in mice, 
a beneficial role of neutrophils was reported during the early lung stages, where neutrophils and macrophages collaborate in the immobilization and death of these parasites, however, this can also lead to increased tissue damage. ${ }^{53}$ But the presence of IL-4 and IL-13 dampens neutrophil activity and thus prevents excessive tissue damage. ${ }^{52}$ Additionally, eosinophils are the main effector cells, downstream of Th2 activation, in immune defense against helminths, thus their increase in the spleen of immunized mice reflects efficient activation of antiparasite Th2 responses. ${ }^{53}$

The most striking aspect of the immune response induced by the OMV:rSmTSP-2 complex is the very high level of specific antibodies, reaching 100-fold higher titers as compared to the rSmTSP-2 protein administered with Alum. In addition, the $\operatorname{IgG} 1: \operatorname{IgG} 2 \mathrm{c}$ ratio shows a switch from a strongly Th2-predominant response induced by rSmTSP-2 to a more balanced Th1/Th2 immune response induced by the complex, correlating with the increased cellular response observed. IgG1 and $\operatorname{IgG} 2 \mathrm{c}$ isotypes have been shown to play a central role in the protective mechanisms mediated by the radiationattenuated cercariae vaccine. ${ }^{30}$ Activation of the two types of $\mathrm{T}$ help can be pivotal to increase the protection against $S$. mansoni infection, in which both types of immune responses are necessary. ${ }^{30}$ Further investigation will determine if this increase and broadening of the immune response will in fact improve protection against infection. If this is confirmed for the SmTSP-2 antigen, other identified targets or key epitopes in the host-parasite interface ${ }^{54}$ can be included in this new antigen-presenting platform.

\section{Conclusion}

This study shows that coupling a recombinant antigen to OMV, using the biotin:rhizavidin affinity interaction, induces a marked increase in the specific immune response to the antigen, both in terms of specific antibody production and in terms of cell-mediated immunity, in comparison with immunity induced by the antigen alone. This underlines the advantages of antigen presentation by a particulate carrier, for better recognition by antigen-presenting cells and also in terms of adjuvant capacity. Since the biotinylated OMV platform allows for coupling of multiple recombinant antigens in fusion with rhizavidin, this opens the perspective of developing multifunctional vaccines, able to induce a broad protection against different pathogens.

\section{Acknowledgments}

This work was supported by FAPESP grant 2017/24632-6 and Fundação Butantan to LCCL and by fellowships from Coordenação de Aperfeiçoamento de Pessoal de Nível Superior Brazil (CAPES) - Finance Code 001 to MMFB and JLS, Conselho Nacional de Desenvolvimento Científico e Pesquisa (CNPq) 160861/2017-9 to MIK. DB was supported by the Italy-Brazil Joint Laboratories initiative of the CNR. We are grateful to Dr. Fabio Rocha Formiga (Instituto Aggeu Magalhães - FIOCRUZ) for his assistance in dynamic light scattering data interpretation. The authors would like to thank the "Serviço de Microscopia Eletrônica" from FIOCRUZ/BA for support, and the facilities of the CIENAM/UFBA for size and zeta potential measurements.

\section{Disclosure}

The authors MMFB, GB and LCCL have a patent application involving the OMV-Antigen complex. The author RM has several patent applications involving the MAPS technology and is a scientific founder, consultant and board member of Affinivax, a biotechnology company that is developing vaccines using the MAPS technology. Ms Violeta Pancakova report grants from EACEA (Education, Audiovisual and Culture Executive Agency) during the conduct of the study; Dr Richard Malley reports personal fees from Merck Vaccines, grants from Pfizer, grants from Astellas, outside the submitted work; Dr Luciana C C Leite reports grants from FAPESP, non-financial support from Fundação Butantan, a Fellowship from $\mathrm{CNPq}$, during the conduct of the study. The authors report no other conflicts of interest in this work.

\section{References}

1. Tebeje BM, Harvie M, You H, Loukas A, McManus DP. Schistosomiasis vaccines: where do we stand? Parasit Vectors. 2016;9(1):528. doi:10.1186/s13071-016-1799-4

2. Fenwick A, Webster JP. Schistosomiasis: challenges for control, treatment and drug resistance. Curr Opin Infect Dis. 2006;19(6):577-582. doi:10.1097/01.qco.0000247591.13671.6a

3. McManus DP, Loukas A. Current status of vaccines for schistosomiasis. Clin Microbiol Rev. 2008;21(1):225-242. doi:10.1128/CMR.00046-07

4. Tendler M, Brito CA, Vilar MM, et al. A Schistosoma mansoni fatty acid-binding protein, $\mathrm{Sm} 14$, is the potential basis of a dual-purpose anti-helminth vaccine. Proc Natl Acad Sci. 1996;93(1):269-273. doi:10.1073/pnas.93.1.269

5. Cardoso FC, Macedo GC, Gava E, et al. Schistosoma mansoni tegument protein Sm29 is able to induce a Th1-type of immune response and protection against parasite infection. PLoS Negl Trop Dis. 2008;2 (10):e308. doi:10.1371/journal.pntd.0000308

6. Ahmad G, Zhang W, Torben W, et al. Protective and antifecundity effects of Sm-p80-based DNA vaccine formulation against Schistosoma mansoni in a nonhuman primate model. Vaccine. 2009;27(21):2830-2837. doi:10.1016/j.vaccine.2009.02.096 
7. Tran MH, Pearson MS, Bethony JM, et al. Tetraspanins on the surface of Schistosoma mansoni are protective antigens against schistosomiasis. Nat Med. 2006;12(7):835-840. doi:10.1038/nm1430

8. Correa-Oliveira R, Pearce EJ, Oliveira GC, et al. The human immune response to defined immunogens of Schistosoma mansoni: elevated antibody levels to paramyosin in stool-negative individuals from two endemic areas in Brazil. Trans $R$ Soc Trop Med Hyg. 1989;83 (6):798-804. doi:10.1016/0035-9203(89)90334-9

9. Fonseca CT, Oliveira SC, Alves CC. Eliminating schistosomes through vaccination: what are the best immune weapons? Front Immunol. 2015;6. doi:10.3389/fimmu.2015.00095.

10. Wilson RA, Li XH, Castro-Borges W. Schistosome vaccines: problems, pitfalls and prospects. Emerg Top Life Sci. 2017;1(6):641-650. doi:10.1042/ETLS20170094.

11. Egesa M, Hoffmann KF, Hokke $\mathrm{CH}$, Yazdanbakhsh M, Cose S. Rethinking schistosomiasis vaccine development: synthetic vesicles. Trends Parasitol. 2017;33(12):918-921. doi:10.1016/j.pt.2017.07.007

12. Reed S, Orr MT, Coler RN. The Vaccines: Vaccine's Adjuvants. Elsevier; 2016. doi:10.1016/C2014-0-00804-0

13. Trovato M. Novel antigen delivery systems. World J Virol. 2015;4 (3):156. doi:10.5501/wjv.v4.i3.156

14. Darrieux M, Goulart C, Briles D, LC de C L. Current status and perspectives on protein-based pneumococcal vaccines. Crit Rev Microbiol. 2015;41(2):190-200. doi:10.3109/1040841X.2013.813902

15. Tan K, Li R, Huang X, Liu Q. Outer membrane vesicles: current status and future direction of these novel vaccine adjuvants. Front Microbiol. 2018;9. doi:10.3389/fmicb.2018.00783.

16. Li R, Liu Q. Engineered bacterial outer membrane vesicles as multifunctional delivery platforms. Front Mater. 2020;7. doi:10.3389/ fmats.2020.00202.

17. Kulp A, Kuehn MJ. Biological functions and biogenesis of secreted bacterial outer membrane vesicles. Annu Rev Microbiol. 2010;64 (1):163-184. doi:10.1146/annurev.micro.091208.073413

18. Santolaya ME, O’Ryan ML, Valenzuela MT, et al. Immunogenicity and tolerability of a multicomponent meningococcal serogroup $\mathrm{B}$ (4CMenB) vaccine in healthy adolescents in Chile: a phase $2 \mathrm{~b} / 3$ randomised, observer-blind, placebo-controlled study. Lancet. 2012;379(9816):617-624. doi:10.1016/S0140-6736(11)61713-3

19. De Temmerman M-L, Rejman J, Demeester J, Irvine DJ, Gander B, De Smedt SC. Particulate vaccines: on the quest for optimal delivery and immune response. Drug Discov Today. 2011;16(13-14):569-582. doi:10.1016/j.drudis.2011.04.006

20. Sahdev P, Ochyl LJ, Moon JJ. Biomaterials for nanoparticle vaccine delivery systems. Pharm Res. 2014;31(10):2563-2582. doi:10.1007/ s11095-014-1419-y

21. Helppolainen SH, Nurminen KP, Määttä JAE, et al. Rhizavidin from Rhizobium etli: the first natural dimer in the avidin protein family. Biochem J. 2007;405(3):397-405. doi:10.1042/BJ20070076

22. Zhang F, Lu Y-J, Malley R. Multiple antigen-presenting system (MAPS) to induce comprehensive B- and T-cell immunity. Proc Natl Acad Sci. 2013;110(33):13564-13569. doi:10.1073/pnas.13072 28110

23. Santos S, Arauz LJD, Baruque-Ramos J, et al. Outer membrane vesicles (OMV) production of Neisseria meningitidis serogroup $\mathrm{B}$ in batch process. Vaccine. 2012;30(42):6064-6069. doi:10.1016/j. vaccine.2012.07.052

24. Frasch CE, van Alphen L, Holst J, Poolman JT, Rosenqvist E. Outer membrane protein vesicle vaccines for meningococcal disease. In: Pollard AJ, Maiden MC editors. Meningococcal Vaccines. Methods in Molecular Medicine. Vol. 66. Humana Press; 2001:81-107. doi:10.1385/1-59259-148-5:81

25. Chromý V, Vinklárková B, Šprongl L, Bittová M. The Kjeldahl method as a primary reference procedure for total protein in certified reference materials used in clinical chemistry. I. a review of Kjeldahl methods adopted by laboratory medicine. Crit Rev Anal Chem. 2015;45(2):106-111. doi:10.1080/10408347.2014.892820
26. Barbosa MMF, Kanno AI, Pancakova V, et al. Expression and purification of Schistosoma mansoni antigens in fusion with rhizavidin for vaccine development. Mol Biotechnol. 2021:1-9. doi:10.1007/ s12033-021-00355-2.

27. Madaan A, Verma R, Singh AT, Jain SK, Jaggi M. A stepwise procedure for isolation of murine bone marrow and generation of dendritic cells. J Biol Methods. 2014;1(1):e1. doi:10.14440/jbm.20 14.12

28. Rose S, Misharin A, Perlman H. A novel Ly6C/Ly6G-based strategy to analyze the mouse splenic myeloid compartment. Cytom Part A. 2012;81A(4):343-350. doi:10.1002/cyto.a.22012

29. Frasch CE, van Alphen L, Holst J, Poolman JT, Rosenqvist E. Outer membrane protein vesicle vaccines for meningococcal disease. In: Meningococcal Vaccines. Vol. 460. New Jersey: Humana Press;2009:81-107. doi:10.1385/1-59259-148-5:81

30. Farias LP, Vitoriano-Souza J, Cardozo LE, et al. Systems biology analysis of the radiation-attenuated schistosome vaccine reveals a role for growth factors in protection and hemostasis inhibition in parasite survival. Front Immunol. 2021;12. doi:10.3389/fimmu. 2021.624191

31. Plotkin SA, Plotkin SL. The development of vaccines: how the past led to the future. Nat Rev Microbiol. 2011;9(12):889-893. doi:10.1038/nrmicro2668

32. Wang S, Huang W, Li K, et al. Engineered outer membrane vesicle is potent to elicit HPV16E7-specific cellular immunity in a mouse model of TC-1 graft tumor. Int $J$ Nanomedicine. 2017;12: 6813-6825. doi:10.2147/IJN.S143264

33. Beernink PT, Vianzon V, Lewis LA, Moe GR, Granoff DM. A meningococcal outer membrane vesicle vaccine with overexpressed mutant FHbp elicits higher protective antibody responses in infant rhesus macaques than a licensed serogroup B vaccine. MBio. 2019;10(3). doi:10.1128/mBio.01231-19

34. Liao TYA, Lau A, Joseph S, Hytönen V, Hmama Z. Improving the Immunogenicity of the Mycobacterium bovis BCG vaccine by non-genetic bacterial surface decoration using the avidin-biotin system. PLoS One. 2015;10(12):1-25. doi:10.1371/journal.pone.014 5833

35. van der Pol L, Stork M, van der Ley P. Outer membrane vesicles as platform vaccine technology. Biotechnol J. 2015;10(11):1689-1706. PMID: 26912077; PMCID: PMC4768646. doi:10.1002/biot.201400395

36. Wai SN, Lindmark B, Söderblom T, et al. Vesicle-mediated export and assembly of pore-forming oligomers of the enterobacterial ClyA cytotoxin. Cell. 2003;115(1):25-35. doi:10.1016/S0092-8674(03) 00754-2

37. Kim J-Y, Doody AM, Chen DJ, et al. Engineered bacterial outer membrane vesicles with enhanced functionality. $J \mathrm{Mol}$ Biol. 2008;380(1):51-66. doi:10.1016/j.jmb.2008.03.076

38. Selvamani V. Stability studies on nanomaterials used in drugs. In: Characterization and Biology of Nanomaterials for Drug Delivery. Elsevier; 2019:425-444. doi:10.1016/B978-0-12-814031-4.00015-5

39. de Jong EC, Smits HH, Kapsenberg ML. Dendritic cell-mediated T cell polarization. Springer Semin Immunopathol. 2005;26 (3):289-307. doi:10.1007/s00281-004-0167-1

40. Blanco P, Palucka A, Pascual V, Banchereau J. Dendritic cells and cytokines in human inflammatory and autoimmune diseases. Cytokine Growth Factor Rev. 2008;19(1):41-52. doi:10.1016/j.cytogfr.2007.10.004

41. Pearson MS, Pickering DA, McSorley HJ, et al. Enhanced protective efficacy of a chimeric form of the schistosomiasis vaccine antigeN Sm-TSP-2. PLoS Negl Trop Dis. 2012;6(3):e1564. doi:10.1371/journal.pntd.0001564

42. Diehl S, Rincón M. The two faces of IL-6 on Th1/Th2 differentiation. Mol Immunol. 2002;39(9):531-536. doi:10.1016/S0161-5890(02) 00210-9

43. Lu Y-J, Gross J, Bogaert D, et al. Interleukin-17A mediates acquired immunity to pneumococcal colonization. PLoS Pathog. 2008;4(9): e1000159. doi:10.1371/journal.ppat.1000159 
44. Zhang F, Ledue O, Jun M, Goulart C, Malley R, Lu Y-J. Protection against staphylococcus aureus colonization and infection by B- and T-Cell-mediated mechanisms. Klugman KP ed. MBio. 2018;9(5). doi:10.1128/mBio.01949-18.

45. Warfel JM, Merkel TJ. The baboon model of pertussis: effective use and lessons for pertussis vaccines. Expert Rev Vaccines. 2014;13 (10):1241-1252. doi:10.1586/14760584.2014.946016

46. Wen X, He L, Chi Y, et al. Dynamics of Th17 cells and their role in schistosoma japonicum infection in C57BL/6 mice. PLoS Negl Trop Dis. 2011;5(11):e1399. doi:10.1371/journal.pntd.0001399

47. Wang B, Norbury CC, Greenwood R, Bennink JR, Yewdell JW, Frelinger JA. Multiple paths for activation of naive CD8 + T cells: CD4-independent help. J Immunol. 2001;167(3):1283-1289. doi:10. 4049/jimmunol.167.3.1283

48. Sa Q, Woodward J, Suzuki Y. IL-2 produced by CD8 + immune $\mathrm{T}$ cells can augment their IFN- $\gamma$ production independently from their proliferation in the secondary response to an intracellular pathogen. J Immunol. 2013;190(5):2199-2207. doi:10.4049/jimmunol.1202256

49. Cosma G, Eisenlohr L. CD8 + T-cell responses in vaccination: reconsidering targets and function in the context of chronic antigen stimulation. F1000Res. 2018;7:F1000Faculty Rev-508. doi:10.126 88/f1000research.14115.1
50. Luis Muñoz-Carrillo J, Francisco Contreras-Cordero J, GutiérrezCoronado O, Trinidad Villalobos-Gutiérrez P, Guillermo RamosGracia L, Elizabeth Hernández-Reyes V. Cytokine profiling plays a crucial role in activating immune system to clear infectious pathogens. In Immune Response Activation and Immunomodulation. IntechOpen; 2019. doi10.5772/intechopen.80843

51. Lloyd CM, Snelgrove RJ. Type 2 immunity: expanding our view. Sci Immunol. 2018;3(25):eaat1604. doi:10.1126/sciimmunol.aat1604

52. Egholm C, Heeb LEM, Impellizzieri D, Boyman O. The regulatory effects of interleukin-4 receptor signaling on neutrophils in type 2 immune responses. Front Immunol. 2019;10. doi:10.3389/fimmu. 2019.02507.

53. Bonne-Année S, Kerepesi LA, Hess JA, et al. Human and mouse macrophages collaborate with neutrophils to kill larval strongyloides stercoralis. Infect Immun. 2013;81(9):3346-3355. doi:10.1128/IAI. 00625-13

54. Farias LP, Vance GM, Coulson PS, et al. Epitope mapping of exposed tegument and alimentary tract proteins identifies putative antigenic targets of the attenuated schistosome vaccine. Front Immunol. 2021;11. doi:10.3389/fimmu.2020.624613

\section{Publish your work in this journal}

The International Journal of Nanomedicine is an international, peerreviewed journal focusing on the application of nanotechnology in diagnostics, therapeutics, and drug delivery systems throughout the biomedical field. This journal is indexed on PubMed Central, MedLine, CAS, SciSearch ${ }^{\mathbb{R}}$, Current Contents ${ }^{\mathbb{R}} /$ Clinical Medicine, $^{-}$
Journal Citation Reports/Science Edition, EMBase, Scopus and the Elsevier Bibliographic databases. The manuscript management system is completely online and includes a very quick and fair peer-review system, which is all easy to use. Visit http://www.dovepress.com/ testimonials.php to read real quotes from published authors. 